\title{
Toxoplasmose: Soroprevalência em Puérperas Atendidas pelo Sistema Único de Saúde
}

Toxoplasmosis: Seroprevalence in Postpartum Women Attended by SUS (Brazilian Public Health System)

Paulo Roberto Dutra Leão, José Meirelles Filho, Sebastião Freitas de Medeiros

\section{RESUM0}

\begin{abstract}
Objetivo: estabelecer a taxa de soroprevalência para toxoplasmose em puérperas atendidas pelo SUS, em duas maternidades de Cuiabá, buscando relacioná-la à idade, antecedentes de abortamento e ao conhecimento desta doença pelas mulheres.

Métodos: trata-se de estudo de corte transversal, sendo a amostra constituída por $205 \mathrm{mu}$ lheres, com idade entre 14 e 43 anos, no primeiro ou segundo dias de puerpério, atendidas nestes hospitais durante dois meses. Cada mulher foi entrevistada através de um pequeno questionário e submetida à coleta de amostra de sangue periférico. Estas amostras foram armazenadas $\mathrm{a}-20^{\circ} \mathrm{C}$ até serem analisadas. A soroprevalência foi determinada, quantitativamente, pela detecção de imunoglobulina $G$ específica contra toxoplasma, através de um método imunoenzimático indireto. Todas as amostras foram analisadas ao mesmo tempo.

Resultados: a média de idade das pacientes foi de 22,4 anos, tendo em média 2,2 gestações, sendo a maioria primigestas. A soropositividade para toxoplasma foi detectada em 165 $(70,7 \%)$ das 205 puérperas estudadas. Não se identificou associação significante entre soropositividade para toxoplasmose e a idade das mulheres $(p=0,967)$ ou o abortamento prévio $(p=0,82)$. A maioria das mulheres incluidas neste estudo desconhecia a existência da toxoplasmose (78\%) e não se identificou associação significante entre este aspecto e maior soroprevalência contra a doença $(p=0,49)$.

Conclusões: esta alta prevalência encontrada é concordante com muitos estudos nacionais e estrangeiros. Permanece, entre o grupo de mulheres incluidas neste estudo, um importante contingente delas $(29,3 \%)$ sob risco de contaminação. A ausência de associação significante entre soroprevalência e a idade das pacientes, relato de abortamento prévio e conhecimento ou não da doença é concordante com alguns estudos e discordante de outros.
\end{abstract}

PALAVRAS-CHAVE: Infecções na gravidez. Toxoplasmose. Transmissão vertical. Soroprevalência. Gravidez. Puerpério.

\section{Introdução}

A toxoplasmose é doença parasitária causada por um protozoário, o Toxoplasma gondii, que acomete o homem e outros animais, tem transcurso geralmente benigno e, pelo risco de abor-

Departamento de Ginecologia da Faculdade de Ciências Médicas da Universidade Federal de Mato Grosso; Departamento de Ginecologia e Obstetrícia da Faculdade de Medicina da Universidade de Cuiabá

Correspondência:

Paulo Roberto Dutra Leão

Rua Brigadeiro Eduardo Gomes, 449 - apto 801 - Goiabeiras

78045-350 - Cuiabá - MS

Fax: (65) 622-0061

e-mail: prdleao@terra.com.br tamentos e transmissão fetal, assume enorme importância quando ocorre em gestante. O parasita reproduz-se de forma assexuada e sexuada nos felinos, seus hospedeiros definitivos, e apenas de forma assexuada nos hospedeiros intermediários, como o homem ${ }^{1}$. No feto, a toxoplasmose manifesta-se por retinocoroidite, micro ou macroencefalia, calcificações cerebrais e retardo mental. Estrabismo e convulsões podem ser observadas. A doença resulta em maior gravidade para o concepto quando acomete a gestante no primeiro trimestre; ocorrendo no último trimestre da gestação, o que acontece em 59 a 65\% dos casos, a criança pode nascer normal ou com manifestações menos importantes ${ }^{2}$. Sabe-se que 
medicação antitoxoplasma, ministrada à mãe, diminui a chance de transmissão para o concepto ${ }^{3}$. Esquema de prevenção, diagnóstico e tratamento antenatal da toxoplasmose congênita com espiramicina para reduzir a transmissão vertical ou pirimetamina associada à sulfadiazina para os fetos infectados, foram propostos há cerca de 15 anos $^{2}$, permanecendo como a melhor abordagem até o momento ${ }^{4}$. O risco de infecção fetal, em qualquer local, está diretamente relacionado à prevalência da doença na população e ao número de mulheres em idade fértil ainda não acometidas e passiveis de contraí-la no decorrer de uma gestação.

A incidência de toxoplasmose congênita é pouco conhecida em nosso meio. Prevalência de toxoplasmose congênita de 1 entre 110 partos, com apenas 50\% dos partos resultando em nativivos, foi descrita em Goiânia ${ }^{5}$. Utilizando os números obtidos nesse trabalho pode-se inferir incidência de aproximadamente 5 por 1000 nascidos vivos, naquela cidade. Estudo realizado no Rio Grande do Sul em 2003 encontrou incidência de 8 casos para 10.000 nascidos vivos ${ }^{6}$. Modelo matemático, desenvolvido em São Paulo, aponta incidência de toxoplasmose congênita de 0,8 para 1000 nascimentos, o que significaria 280 casos novos por ano, naquela cidade ${ }^{7}$. Estabelecendo-se a prevalência desta parasitose em dada população, pode-se ter idéia da probabilidade de contaminação dos indivíduos e também do número de indivíduos suscetíveis a esta contaminação. Esta prevalência é, portanto, fundamental para medidas que permitam diminuir a contaminação e minimizar as seqüelas da doença na população mais vulnerável. A importância de se estabelecer o perfil sorológico da mulher em idade reprodutiva reside na possibilidade de se tomarem medidas terapêuticas para minimizar a transmissão vertical, na vigência de infecção aguda na gravidez, e de se fazer seguimento sorológico e orientação higienodietética pré-natal adequada para a paciente suscetível, para evitar a sua contaminação ${ }^{8}$. Este estudo tem como proposta estabelecer a prevalência da toxoplasmose em amostra de puérperas atendidas pelo SUS, nas principais maternidades de Cuiabá, e identificar o perfil epidemiológico para toxoplasmose, na população estudada.

\section{Pacientes e Métodos}

Estudo de corte transversal, no qual se analisa a prevalência de anticorpos da classe imunoglobulina G contra Toxoplasma gondii, no soro de um grupo de 205 mulheres em puerpério imediato, atendidas pelo SUS, em duas maternidades de Cuiabá, Mato Grosso. Foram consideradas elegíveis puérperas, entre o primeiro e o terceiro dias pós-parto, internadas nos hospitais Universitário Júlio Müller e Hospital Geral Universitário, nos meses de abril e maio de 2000 e aquelas que anuíram em participar do estudo. Foram excluídas mulheres em uso de imunossupressores e aquelas que não concordaram em assinar o termo de consentimento livre e esclarecido. A idade média das pacientes incluídas foi de $22,4 \pm 5,6$ anos, tendo a mais jovem 14 anos e a mais idosa 43 , sendo que $35 \%$ tinham menos de 20 anos.

O tamanho amostral foi calculado considerando-se o desenho do estudo, taxa de prevalência de sorologia positiva de $77 \%{ }^{9}$, precisão absoluta de $6,5 \%$ e nível de confiança de $95 \%$. Consideraram-se para análise as variáveis: número de gestações, resultados gestacionais anteriores e faixa etária. O contato preliminar, a obtenção do termo de consentimento, a entrevista para coleta de dados e a coleta de amostras de sangue foram precedidos dos esclarecimentos pertinentes.

Foram colhidas amostras de $5 \mathrm{~mL}$ de sangue por punção de veia periférica. O sangue de cada amostra foi deixado coagular à temperatura ambiente e centrifugado por 15 minutos, acondicionado para armazenagem, em tubo identificado, a $-20^{\circ} \mathrm{C}$ até a realização dos testes sorológicos. Todas as determinações foram realizadas ao mesmo tempo, utilizando-se ensaio imunoenzimático indireto para determinar o título de anticorpos, da classe IgG, contra Toxoplasma gondii, em soro humano (Cobas Core Toxo IgG EIA II, fabricado por F. Hoffman, La Roche Ltda., Basel, Suíça, e distribuído no Brasil pela Roche Farmacêutica Buímica Ltda., São Paulo).

Os dados do estudo foram analisados pelo programa Epi-Info, versão 6.04a ${ }^{10}$. A comparação entre proporções foi efetuada pelo teste do $\chi^{2}$, aceitando-se diferenças com significância estatística quando $p \leq 0,05$. Este estudo seguiu os preceitos éticos contidos na resolução número 196/96 do Conselho Nacional de Saúde (Brasil, 1996), que normatiza pesquisas envolvendo seres humanos, e foi aprovado pelo Comitê de Ética em Pesquisa do Hospital Universitário Júlio Muller, da Universidade Federal de Mato Grosso.

\section{Resultados}

A maioria das pacientes era procedente de Cuiabá $(67,3 \%)$ e Várzea Grande (23,9\%); a quase 
totalidade das demais residia em cidades circunvizinhas. Incluindo-se a gestação atual, as puérperas que fizeram parte do estudo tinham em média $2,2 \pm 1,3$ gestações, sendo $40 \%$ primigestas, $25 \%$ secundigestas e $21 \%$ tercigestas. História de um ou mais abortamentos anteriores foi relatada por 36 pacientes $(17,5 \%)$. Cerca de $94,1 \%$ afirmaram ter recebido atendimento de pré-natal, tendo o número de consultas variado entre 2 e 9 . Entre estas pacientes, $61,0 \%$ tiveram as seis consultas estabelecidas como mínimo pela Organização Mundial de Saúde.

Apenas 21,9\% das pacientes na população estudada referiram conhecer toxoplasmose, sendo que as demais $(78,1 \%)$ afirmaram jamais ter ouvido falar desta doença (Tabela 1). Dentre as 205 puérperas estudadas, $145(70,7 \%)$ tiveram resultado positivo para imunoglobulina G antitoxoplasma e, nas 60 restantes $(29,3 \%)$, a sorologia foi negativa.

Tabela 1 - Associação entre conhecimento da paciente sobre a doença e sorologia para toxoplasmose.

\begin{tabular}{|c|c|c|c|c|c|c|}
\hline \multirow[t]{2}{*}{ Conhece toxoplasmose } & \multicolumn{2}{|c|}{ Sorologia positiva } & \multicolumn{2}{|c|}{ Sorologia negativa } & \multicolumn{2}{|c|}{ Total } \\
\hline & $\mathrm{n}$ & $\%$ & $\mathbf{n}$ & $\%$ & $\mathbf{n}$ & $\%$ \\
\hline Sim & 30 & 66,7 & 15 & 33,3 & 45 & 21,9 \\
\hline Não & 115 & 71,8 & 45 & 28,2 & 160 & 78,1 \\
\hline Total & 145 & 70,7 & 60 & 29,3 & 205 & 100 \\
\hline
\end{tabular}

Quando se analisou a idade das pacientes em confronto com a presença de sorologia positiva para toxoplasmose, não se encontrou associação significante entre os dados obtidos, embora houvesse ligeira elevação da prevalência nas pacientes com idade superior a 30 anos (Tabela 2).
Com relação ao abortamento em gestação anterior e soropositividade para toxoplasmose, de 36 pacientes com história obstétrica de abortamento, 26 tinham sorologia positiva, perfazendo prevalência de $72,2 \%$, contra $70,4 \%$ encontrados no grupo sem tal antecedente (Tabela 3).

Tabela 2 - Associação entre idade das pacientes e sorologia para toxoplasmose.

\begin{tabular}{lccccr}
\hline Idade & \multicolumn{2}{c}{ Sorologia positiva } & \multicolumn{2}{c}{ Sorologia negativa } & \multicolumn{2}{c}{ Total } \\
& $\mathbf{n}$ & $\%$ & $\mathbf{n}$ & $\%$ & $\mathbf{n}$ \\
\hline$<20$ anos & 51 & 70,8 & 21 & 29,2 & 72 \\
20 a 25 anos & 52 & 68,4 & 24 & 31,6 & 35,1 \\
25 a 30 anos & 23 & 71,9 & 9 & 28,1 & 37,1 \\
30 a 35 anos & 13 & 76,4 & 4 & 23,6 & 32 \\
$>35$ anos & 6 & 75,0 & 2 & 25,0 & 15,6 \\
Total & 145 & 70,7 & 60 & 29,3 & 8,3 \\
\hline
\end{tabular}

$\chi^{2}=0,56 ; p=0,967$.

Tabela 3 - Antecedentes de abortamento em gestações anteriores e sorologia para toxoplasmose.

\begin{tabular}{|c|c|c|c|c|c|c|}
\hline \multirow[t]{2}{*}{ Antecedentes de abortamento } & \multicolumn{2}{|c|}{ Sorologia positiva } & \multicolumn{2}{|c|}{ Sorologia negativa } & \multicolumn{2}{|c|}{ Total } \\
\hline & $\mathrm{n}$ & $\%$ & $\mathrm{n}$ & $\%$ & $\mathrm{n}$ & $\%$ \\
\hline Sim & 26 & 72,3 & 10 & 27,8 & 36 & 17,6 \\
\hline Não & 119 & 70,4 & 50 & 29,6 & 169 & 82,4 \\
\hline Total & 145 & 70,7 & 60 & 29,3 & 205 & 100 \\
\hline
\end{tabular}




\section{Discussão}

Neste estudo fez-se levantamento do estado sorológico contra toxoplasmose em população de puérperas de Cuiabá, atendidas pelo Sistema Único de Saúde (SUS), em duas maternidades locais, responsáveis por $48,5 \%$ dos nascimentos nesta cidade (Sistema de Informação de Nascimentos da Fundação de Saúde de Cuiabá, SINASC - FUSC, 1997). A amostra foi retirada da população de pacientes atendidas por estes hospitais, nos meses de abril e maio de 2000. Guanto à idade e paridade, o perfil encontrado é semelhante ao descrito pela maioria dos estudos nacionais envolvendo mulheres em idade reprodutiva ${ }^{11-13}$.

Aspecto interessante, e pouco citado na literatura, é o conhecimento da doença por parte das pacientes. Duarte et al. ${ }^{12}$, em estudo realizado com puérperas em Ribeirão Preto (São Paulo), constataram que $86 \%$ delas desconheciam a doença. Já em estudo realizado nos Estados Unidos da América, os autores constataram que 48\% das mulheres já tinham algum tipo de informação sobre a toxoplasmose ${ }^{13}$. No presente estudo esta taxa de desconhecimento da parasitose atingiu índice semelhante à cifra nacional citada, já que $78,1 \%$ das pacientes, quando perguntadas, referiram desconhecer a existência da toxoplasmose.

A prevalência de soropositividade para imunoglobulina G $(70,7 \%)$ situa-se dentro dos resultados já obtidos em outros estudos nacionais, envolvendo populações de gestantes ou puérperas. Em estudo transversal realizado com gestantes atendidas em duas maternidades de Salvador, Moreira ${ }^{14}$ encontrou soroprevalência para toxoplasma de $42 \%$, sendo a menor cifra relatada no Brasil. Taxa de soroprevalência em gestantes de $57,6 \%$ foi encontrada em Uberlândia, Minas Gerais $^{11}$, e Duarte et al. ${ }^{12}$, em estudo de prevalência sorológica, realizado com puérperas atendidas no Hospital das Clínicas (USP) de Ribeirão Preto (São Paulo), chegaram a resultado de 61\% de positividade. Estudo realizado no Rio Grande do Sul, em população de gestantes, encontrou soroprevalência para toxoplasma de $74,5 \%{ }^{15}$. Meirelles-Filho ${ }^{9}$, em 1985, em estudo transversal realizado com gestantes na cidade do Rio de Janeiro, encontrou taxa de prevalência de $77,5 \%$ para toxoplasmose. A maior cifra relatada em estudos nacionais foi de $78,5 \%$, em estudo de prevalência realizado com gestantes de um serviço de pré-natal, também no Rio de Janeiro ${ }^{16}$. Neves et al. ${ }^{17}$ encontraram positividade de $54,3 \%$ em gestantes de Porto Alegre, e Pedreira ${ }^{8}$, de 58,8\%, em gestantes de São Paulo.
Em termos mundiais, as taxas de prevalência em gestantes variam de acordo com o perfil do país estudado, encontrando-se índices menores (11 a 14\%) em países escandinavos ${ }^{18,19}$. Índices maiores foram observados em $51,0 \%$ de puérperas na França ${ }^{20}$, em 55,7\% de gestantes na Malásia ${ }^{21}$, em 70,9\% das gestantes em $\mathrm{Cuba}^{22}$ e em $80,5 \%$ de puérperas na Nigéria ${ }^{23}$. Nos países vizinhos ao Brasil, destacam-se as taxas de prevalência de $39,3 \%{ }^{24}$ e de $58,9 \%{ }^{25}$ relatadas em gestantes argentinas.

Acredita-se que quanto mais alta a faixa etária, maior a porcentagem de indivíduos infectados e, portanto, soropositivos ${ }^{26}$. Isto pode ser explicado pelo maior tempo de exposição à doença. Em estudo com gestantes realizado na Nigéria, encontrou-se diferença significativa na soroprevalência para o toxoplasma à medida que aumentava a faixa etária das pacientes, com taxas de $25 \%$ no grupo de 15 a 18 anos e de $71,4 \%$ no grupo de 39 a $42^{27}$. Na Bélgica, encontrou-se também diferença estatisticamente significativa nas taxas de soroprevalência das diversas faixas etárias, em população de gestantes e mulheres doadoras de sangue ${ }^{28}$. Os autores observaram taxas menores entre as pacientes com 20 anos (45\% nas gestantes e $50 \%$ nas doadoras) que entre as pacientes com 40 anos de idade $160 \%$ nas gestantes e $72 \%$ nas doadoras). O estudo conduzido na Noruega por Jenum et al. ${ }^{18}$ também concluiu que houve diferença estatisticamente significativa nas taxas de soroprevalência quando os grupos de gestantes com mais ou menos de 34 anos foram comparados. Há estudos que contradizem esta tendência de aumento da prevalência à medida que avança a idade das pacientes. Lelong et al. ${ }^{29}$, estudando gestantes em Madagascar, não encontraram diferença entre pacientes de diversas faixas etárias (75\% no grupo entre 15 e 19 anos, $81 \%$ no grupo entre 20 e 24 anos e $83,5 \%$ nas pacientes acima destes limites etários). Fuente et al. ${ }^{25}$, 1997, não observaram diferença estatisticamente significativa entre as gestantes argentinas de menos de 20 anos $(58,8 \%)$, entre 20 e 29 anos $(56,9 \%)$ ou entre 30 e 39 anos $(64,2 \%)$. À mesma conclusão chegaram Moreira ${ }^{14}$, em Salvador, e Brisighelli ${ }^{30}$, em Bragança Paulista. No presente estudo não se encontrou diferença significante nas taxas de soroprevalência positiva para toxoplasmose nas diferentes faixas etárias da população estudada $(p=0,967)$

Outro aspecto epidemiológico, objeto de vários estudos, é a maior prevalência de toxoplasmose em pacientes com história obstétrica de abortamentos. Estudo do tipo caso-controle, realizado em Guadalajara, México, evidenciou maior 
prevalência de toxoplasmose no grupo de pacientes com história de abortamento habitual do que no grupo controle ${ }^{31}$. Resultado semelhante foi encontrado por Guerra Garcia e Fernandez Sampedro $^{32}$ em estudo conduzido com puérperas, na cidade de Málaga, Espanha. Outros estudos, realizados em Aman, Jordânia ${ }^{33}$, e em Bragança Paulista $^{30}$, não encontraram associação estatisticamente significativa entre estas duas variáveis. No presente estudo também não se constatou esta associação, com soropositividade de $72,2 \%$ no grupo de pacientes com história de abortamento anterior e de 70,4\% no grupo sem tal antecedente.

Os resultados obtidos no presente estudo identificam a alta prevalência da infecção por toxoplasma nas puérperas de Cuiabá $(70,7 \%)$ e, mais ainda, mostram a existência de importante contingente de mulheres suscetiveis de adquirir a doença no transcurso da gestação. Estas pacientes suscetiveis correm o risco de, por meio da transmissão vertical, transmitir a doença a seus conceptos, causar-lhes danos irreparáveis ou mesmo levá-los ao óbito. A identificação destas pacientes, por meio de exame sorológico no início da gestação, possibilita a adoção de medidas de orientação higieno-dietéticas, no intuito de se evitarem sua contaminação durante a gestação e a possivel transmissão a seus fetos. Pode-se sugerir, portanto, que o rastreamento sorológico sistemático para toxoplasmose, no pré-natal, é medida de saúde pública a ser implementada na comunidade examinada.

\section{ABSTRACT}

Objective: to establish the rate of toxoplasmosis soroprevalence in postpartum women, attended in two hospitals of the Public Health System (SUS) in Cuiabá, and its correlation with age, previous abortion and women's knowledge of the disease.

Methods: a cross-sectional study including 205 women with ages from 14 to 43 (mean 22.4) years old, attended in these hospitals for two months, in the first or second days postpartum. Each woman answered a short questionnaire and had peripheral blood sample collected. Blood samples were stored at $-20^{\circ} \mathrm{C}$ until assay. The seroprevalence was determined by quantitative detection of specific Ig $G$ antibody against Toxoplasma gondii, using a microparticle enzyme immunoassay. All samples were assayed at the same time.

Results: The average number of pregnancies in the women included in this study was 2.2, and the majority of them was pregnant for the first time. The seroprevalence found was $70.7 \%$ (165 of 205 women). No statistical correlation was found between seroprevalence and age $(p=0.967)$ or previous abortion $(p=0.82)$. Most of the women in this study (78\%) did not know about toxoplasmosis and no statistical correlation was found between this condition and seroprevalence $(p=0.49)$.

Conclusions: the high seroprevalence found in the present study is in accordance with surveys previously reported in our country and other developing countries. Among the women included in this study, an expressive number (29.3\%) is still under risk of contamination. The lack of statistical correlation between seroprevalence and women's age, lack of knowledge about the disease and report of previous abortion is in accordance with some previous studies and in disagreement with others.

KEYWORDS: Toxoplasmosis. Seroprevalence. Pregnancy. Postpartum.

\section{Referências}

1. Weiss LM, Kim K. The International Congress on Toxoplasmosis. Int J Parasitol 2004; 34:249-52.

2. Daffos F, Forestier F, Capella-Pavlovsky M, et al. Prenatal management of 746 pregnancies at congenital toxoplasmosis. N Engl J Med 1988; 318:271-5.

3. Desmonts G, Couvreur J. Congenital toxoplasmosis. A prospective study of 378 pregnancies. N Engl J Med 1974; 290:1110-6.

4. Wallon M, Gaucherand P, Al Kurdi M, Peyron F. Toxoplasma infections in early pregnancy: consequences and management. J Gynecol Obstet Biol Reprod (Paris) 2002; 31:478-84.

5. Viggiano MGC, Moraes-Filho JT, Oliveira SA, Amaral WN, Ximenes YR. Toxoplasmose congênita: a segunda infecção mais importante da síndrome Storch. J Bras Ginecol 1991; 10:293-302.

6. Mozzatto L, Procianoy RS. Incidência de toxoplasmose congênita no sul do Brasil: um estudo prospectivo. Rev Inst Med Trop São Paulo 2003; 45:147-51.

7. Guimarães ACS, Kawarabayashi M, Borges MM, Tolezano JE, Andrade-Junior HF. Variações regionais na soronegatividade para toxoplasmose na região metropolitana de São Paulo. Rev Inst Med Trop São Paulo 1993; 35:479-83.

8. Pedreira DAL. Contribuição ao estudo da toxoplasmose congênita [dissertação]. São Paulo: Univ. de São Paulo; 1995.

9. Meirelles-Filho J. Toxoplasmose e gravidez: inquérito sorológico em gestantes e seus recém-nascidos na Maternidade Escola da Universidade Federal do Rio de Janeiro. J Bras Ginecol 1985; 95:393-401. 
10.Dean AG, Dean JA, Coulombier D. Epi-Info version 6: a word-processing, database and statistics program for public health on IBM-compatible microcomputers [computer program]. Atlanta: Center for Diseases Control and Prevention; 1995.

11.Segundo GRS, Silva DAO, Mineo JR, Ferreira MS. Um estudo comparativo de toxoplasmose congênita entre hospitais públicos e privados de Uberlândia, MG, Brasil. Mem Inst Oswaldo Cruz 2004; 99:13-7.

12.Duarte G, Paschoini MC, Martinez R, Ramos DM, Turco F. Soroprevalência para toxoplasmose em puérperas do Hospital das Clínicas da Faculdade de Medicina de Ribeirão Preto, da Universidade de São Paulo. In: Anais do $48^{\circ}$ Congresso Brasileiro de Ginecologia e Obstetrícia; 1999; Goiânia; 1999. [Tema Livre número 126, apresentação oral].

13.Jones JL, Ogunmodede F, Scheftel J, et al. Toxoplasmosis-related knowledge and practices among pregnant women in the United States. Infect Dis Obstet Gynecol 2003; 11:139-45.

14. Moreira LMO. Sorologia para toxoplasmose em uma população de gestantes de Salvador [tese]. Salvador: Univ. Federal da Bahia; 1988.

15.Spalding SM, Amendoeira MRR, Ribeiro LC, Silveira C, Garcia AP, Camillo-Coura L. Estudo prospectivo de gestantes e seus bebês com risco de transmissão de toxoplasmose congênita em Município do Rio Grande do Sul. Rev Soc Bras Med Trop 2003; 36:483-91.

16. Coutinho SG, Souza WJ, Camilo-Coura L, Marzochi MC, Amendoeira MRR. Levantamento dos resultados das reações de imunofluorescência indireta para toxoplasmose em 6079 pacientes de ambulatório ou gestantes no Rio de Janeiro, realizadas durante os anos de 1971 a 1977 . Rev Inst Med Trop São Paulo 1981; 23:48-56.

17.Neves JM, Nascimento LB, Ramos JGL, Martins Costa SH. Toxoplasmose na gestação. Rev Bras Ginecol Obstet 1994; 16:197-202.

18.Jenum PA, Stray-Pedersen B, Melby KK, et al. Incidence of Toxoplasma gondii infection in 35,940 pregnant women in Norway and pregnancy outcome for infected women. J Clin Microbiol 1998; 36:2900-6.

19.Evengard B, Petersson K, Engmar ML, et al. Low incidence of toxoplasma infection during pregnancy and in newborns in Sweden. Epidemiol Infect 2001; 127:121-7.

20.Wallon M, Mallaret MR, Mojon M, Peyron F. Congenital toxoplasmosis, evaluation of the prevention policy. Presse Med 1994; 23:1467-70.
21.Nissapatorn V, Noor Azmi MA, Cho SM, et al. Toxoplasmosis: prevalence and risk factors. J Obstet Gynaecol 2003; 23:618-24.

22. Gonzales-Morales T, Bacallo-Gallestey J, GarciaSantana CA, Molina-Garcia JR. Prevalence of Toxoplasma gondii antibodies in a population of pregnant women in Cuba. Gac Med Mex 1995; 131:499503.

23. Onadeko MO, Joynson DH, Payne RA, Francis J. The prevalence of toxoplasma antibodies in pregnant Nigerian women and the occurrence of stillbirth and congenital malformation. Afr J Med Sci 1996; 25:331-4.

24.Hakin A, Dunaiewsky A, Berenstein F, et al. Toxoplasmosis and Chagas. Serological prevalence in pregnancy in the Ramos Mejia Municipal Hospital. Rev Soc Obstet Ginecol B Aires 1998; 77:273-86.

25.Fuente MC, Bovone NS, Cabral GE. Prophylaxis of prenatal toxoplasmosis. Medicina (B Aires) 1997; 57:155-60.

26. Frenkel JK. Prevention of toxoplasma infection in pregnant women and their fetuses. Clin Infect Dis 1995; 20:727-9.

27. Olusi T, Gross U, Ajayi J. High incidence of toxoplasmosis during pregnancy in Nigeria. Scand J Infect Dis 1996; 28:645-6.

28.Luyasu V, Robert A, Lissenko D, et al. A soroepidemiological study on toxoplasmosis. Acta Clin Belg 1997; 52:68.

29.Lelong B, Rahelimino B, Candolfi E, et al. Prevalence de la toxoplasmose dans une population de femmes enceintes a Tananarive (Madagascar). Bull Soc Pathol Exot 1995; 88:46-9.

30.Brisighelli AN. Prevalência da toxoplasmose em gestantes da cidade de Bragança Paulista - São Paulo [dissertação]. São Paulo: Univ. de São Paulo; 1998.

31.Galván-Ramírez ML, Soto Mancilla JL, Velasco Castrejon O, Pérez Medina R. Incidence of antitoxoplasma antibodies in women with high-risk pregnancy and habitual abortions. Rev Soc Bras Med Trop 1995; 28:333-7.

32.Guerra Garcia C, Fernandez Sampedro J. Seroprevalence of Toxoplasma gondii pregnant women. Aten Primaria 1995; 16:151-3.

33. Qublan HS, Jumaian N, Abu-Salem A, Hamadelil FY, Mashagbeh M, Abdel-Ghani F. Toxoplasmosis and habitual abortion. J Obstet Gynaecol 2002; 22:296-8. 